\title{
Screening and mixture design applied to formulate anti- caking compositions for reducing the caking tendency of granular phosphate fertilizers
}

\author{
Mohammed Lakehal Senhaji ${ }^{1,}$ *, Anass Hafnaoui ${ }^{2}$, Mehdi Khouloud ${ }^{3}$, Asmae El Agri ${ }^{1}$, Mohammed El \\ Asri ${ }^{1,3}$ and Ahmed Boulahna ${ }^{1}$ \\ ${ }^{1}$ Organic Chemistry Labs, FST Fez, USMBA, Po Box. 2202 - Imouzzer Road, Fez, Morocco. \\ ${ }^{2}$ Innovation OCP Group, Jorf Lasfar, Morocco \\ ${ }^{3}$ Chemical \& Biochemical Sciences Laboratory, Mohammed VI Polytechnic University, Lot 660, Hay Moulay \\ Rachid, 43150, Ben Guerir, Morocco
}

\begin{abstract}
The demand for fertilizers in the agricultural sector has increased drastically with the world's exponential growth. Moreover, solid granular fertilizers remain by far the most requested. The granular fertilizers production presents several kinds of problems, especially the ternary fertilizers family. The caking is a severe issue that affects granules' physical quality, particularly during storage and transport. It makes them unsuitable for their intended uses. In this work, a new anti-caking agent has been developed to cope with the granular fertilizer caking problem and improve their physical quality. First of all, thanks to the screening design of Plackett-Burman, the effect of seven anti-caking components on the phosphate fertilizer caking trend was evaluated only by eight trials. Next, a mixture design was used to formulate a new anti-caking agent from three components (glycerol, calcium carbonate, and molasses). The screening design was chosen as the most effective components. Finally, from the mixture design results, a special cubic model was postulated and statically validated. The model delivered a wide range of compositions formulated from the three components mentioned above, capable of reducing the caking tendency of ternary granular fertilizer.
\end{abstract}

Keywords: Screening design; Plackett-Burman design; Mixture design; Anti-caking agents; Caking fertilizers.

\section{Introduction}

Mineral fertilizers contain more concentrated, consistent, and readily available nutrients than organic fertilizers, enabling farmers to grow more on less land. Without them, world agricultural production could fall by as much as half, and it would not be possible to feed the global population, which is perpetually rising. Thus, the world demand for complete fertilizers is estimated to increase with an average of $1.3 \%$ in $2022 / 23$. Moreover, potash and phosphate demand is expected to expand faster than that for nitrogen, by $1.8,1.4$, and $1.0 \%$ during this period $^{1-2}$.

Dry granular fertilizers are widely used because they are less expensive and easier to handle and apply than liquid fertilizers. Their hygroscopic characteristics quickly absorb moisture during storage and transport, accelerating their caking ${ }^{3}$. Caking or agglomeration phenomenon is a serious issue when we handle such fertilizers; therefore, it has been studied for a long time $^{4-5}$, up to now ${ }^{6}$.

*Corresponding author: Mohammed Lakehal Senhaji Email address: lakehalsenhaji.mohammed@gmail.com DOI: $\underline{\text { http://dx.doi.org/10.13171/mjc02102221569mls }}$
It is essential to highlight that caking leads to a great deal of negative impact, especially on products' physical quality. Indeed, the liquid occurring from the absorbed moisture bridges between particles due to capillary condensation ${ }^{7-8}$. This exciting phenomenon leads to solid bridges caused by crystallization of the granules after evaporation of moisture, which brings to the aggregation of fertilizer granules. Keeping the material dry can avoid the caking issue. However, this is unachievable on a large scale. In this case, the grains should be coated with suitable anti-caking agents ${ }^{9-12}$.

Due to their ability to reduce adhesion and ensure the free-flowing of particles, the coating agents containing dry anti-caking powder are widely used in the field of granular fertilizers production ${ }^{13-20}$. They were also used in the food and pharmaceutical industry, such as salts, sugars, shredded cheese, drug powder, etc., ${ }^{21-24}$.

As mentioned above, there are many workers interested in this topic. Also, several products were used as an anti-caking agent. Testing all of them 
requires many experiments; therefore, it is quite clever to use the experiment's design.

Design of Experiment (DoE) is a set of multivariate analysis techniques that aim to acquire the maximum amount of information from an experimental system. The number of experimental runs is small or a minimum ${ }^{25}$. Plackett-Burman Design is regularly used to evaluate the principal effects of many variables while reducing the number of experimental runs. It is generally used to screen the significant variables and to eliminate the irrelevant ones.

For the above reasons, it seems logical to use the Plackett-Burman screening design to select from the list of the given components: sodium carbonate, calcium carbonate, silicon dioxide, sodium sulfate, potassium sulfate, molasses, and glycerol; components that have a significant effect on the cake strength (CS) of ternary granular fertilizer.

The formulation of an anti-caking agent should be done by a mixture of three components (calcium carbonate, molasses, and glycerol) which have been chosen by the screening design as significant components. For this purpose, preparing and modifying mixtures needs the application of another type of DoE ${ }^{26}$. This involves the use of mixture designs to prepare several anti-caking compositions and explore how such arrangements can reduce the cake strength of granules and then improve their physical quality.

\section{Materials and methods}

\subsection{Materials}

Ternary granular fertilizer (NPK 15-15-15, 2-4 mm on diameter) was obtained from OCP Group (Plant of Jorf Lasfar, Morocco). The cake strength (CS) of fertilizer was determined using a Pellet Testing Machine Model PT-II, called a durometer, and manufactured by JT Tohsi Inc. (Japan, Tokyo).

The tested components were: sodium carbonate $\left(\mathrm{Na}_{2} \mathrm{CO}_{3}\right.$, A.R. grade) was purchased from FLUKA (Germany), calcium carbonate $\left(\mathrm{CaCO}_{3}\right.$, A.R. grade) (GB Aromatic Chemicals, UK), silicon dioxide $\left(\mathrm{SiO}_{2}\right.$, A.R. grade) (Merck, Germany), sodium sulfate $\left(\mathrm{Na}_{2} \mathrm{SO}_{4}\right.$, A.R. grade) (Solvachim, Morocco), potassium sulfate $\left(\mathrm{K}_{2} \mathrm{SO}_{4}\right.$, A.R. grade) (Scharlau, Spain), glycerol $\left(\mathrm{C}_{3} \mathrm{H}_{8} \mathrm{O}_{3}\right.$, A.R. grade) (VWR Chemicals, France), and molasses (Cosumar, Morocco). All of these products were available in the laboratory.

Data processing was done using NEMRODW software $^{27}$.

\subsection{Methods}

\subsubsection{Experimental designs}

The experimental design methodology also called the experiment (DoE) design, is an ingenious way to plan, optimize, and control a process ${ }^{28-30}$. It can substantially reduce time, cost and lead to procedures and products that perform better and have higher reliability than those developed using classic experimental approaches. For these reasons, DoE is widely used in many applications and for several objectives $^{28-31}$.

There are many types of experimental designs ${ }^{31-32}$, and each method is used for a specific situation. In this study, we started by Plackett-Burman screening design ${ }^{33-35}$ to evaluate the principal effects of seven compounds on a granular fertilizer's cake strength. This part aims to identify each component's impact for retaining the more efficient and giving up the irrelevant ones.

Hereafter, the screening design's significant components are used to formulate a new anti-caking agent using a mixture experimental design ${ }^{36-40}$. This section focused on studying the variation effect of a three-components system with constraints ${ }^{41-42}$ and understanding how the cake strength of fertilizer is affected by the variation of the proportion of different mixture component fractions.

\subsubsection{Accelerated caking test}

To investigate the fertilizer caking tendency, an accelerated caking test was applied. On a laboratory scale, the method involves accelerating the caking tendency using centrifugation apparatus with cylindrical molds. A fertilizer test sample of $30 \mathrm{~g}$ was placed in the mold, then centrifugation was maintained for 2 hours at a temperature of $25-26^{\circ} \mathrm{C}$. Once the time has elapsed, the mold can be split into two parts so that the cake formed could be removed without any breakage.

\subsubsection{Fertilizer cake strength}

To measure the cake strength, fertilizer samples were screened using a Pellet Testing Machine Model PT-II (durometer). The device was used to measure the mechanical strength of the fertilizer sample after the accelerated caking test. The sample was placed on a flat surface where the pressure was applied by an electric motor driven. A force sensor mounted in the durometer measured the pressure required to fracture the fertilizer sample. The load at which the sample fractured was considered as the cake strength of the tested fertilizer.

\section{Results and discussion}

\subsection{Screening design}

The seven compounds used in this study were mentioned in several types of research ${ }^{43-46}$. Table 1 presents components examined as independent parameters affecting the cake strength of granular phosphate ternary fertilizer and their experimental domain in the Plackett-Burman screening design. 
Table 1. Parameters and their experimental domain in Plackett-Burman screening design.

\begin{tabular}{|c|c|c|c|}
\hline \multirow{2}{*}{ Parameters } & \multirow{2}{*}{ Unit } & \multicolumn{2}{|c|}{ Levels } \\
\cline { 3 - 4 } & & Low level (-1) & High level (+1) \\
\hline $\boldsymbol{X}_{\mathbf{1}}$ : Glycerol & $\%$ & 0.4 & 0.8 \\
\hline $\boldsymbol{X}_{\mathbf{2}}$ : Molasses & $\%$ & 0.4 & 0.8 \\
\hline $\boldsymbol{X}_{\mathbf{3}}$ : Sodium sulfate & $\%$ & 0.2 & 0.4 \\
\hline $\boldsymbol{X}_{\mathbf{4}}$ : Potassium sulfate & $\%$ & 0.2 & 0.4 \\
\hline $\boldsymbol{X}_{\mathbf{5}}$ : Calcium carbonate & $\%$ & 0.2 & 0.4 \\
\hline $\boldsymbol{X}_{\mathbf{6}}$ : Sodium carbonate & $\%$ & 0.2 & 0.4 \\
\hline $\boldsymbol{X}_{\mathbf{7}}$ : Silicon dioxide & $\%$ & 0.2 & 0.4 \\
\hline
\end{tabular}

We have postulated that each experiment's result is a linear combination of the effect of each variable, $X_{l}$, $X_{2}, \ldots \ldots X_{k}$, and that the measured response for each experiment $j$ is $Y_{j}$. The mathematical model for the seven variables is the 1st-degree polynomial model as given by Eq.1:

$Y=b_{0}+b_{1} X_{1}+b_{2} X_{2}+b_{3} X_{3}+b_{4} X_{4}+b_{5} X_{5}+b_{6} X_{6}+b_{7} X_{7}$

The cake strength (CS) results of all experiments were evaluated using a durometer apparatus as mentioned in Figure 1 below. We started testing an uncoated fertilizer used as a reference to assess the efficiency of the tested coating components. The cake strength result found after the test is $39.0 \mathrm{kgf}$.

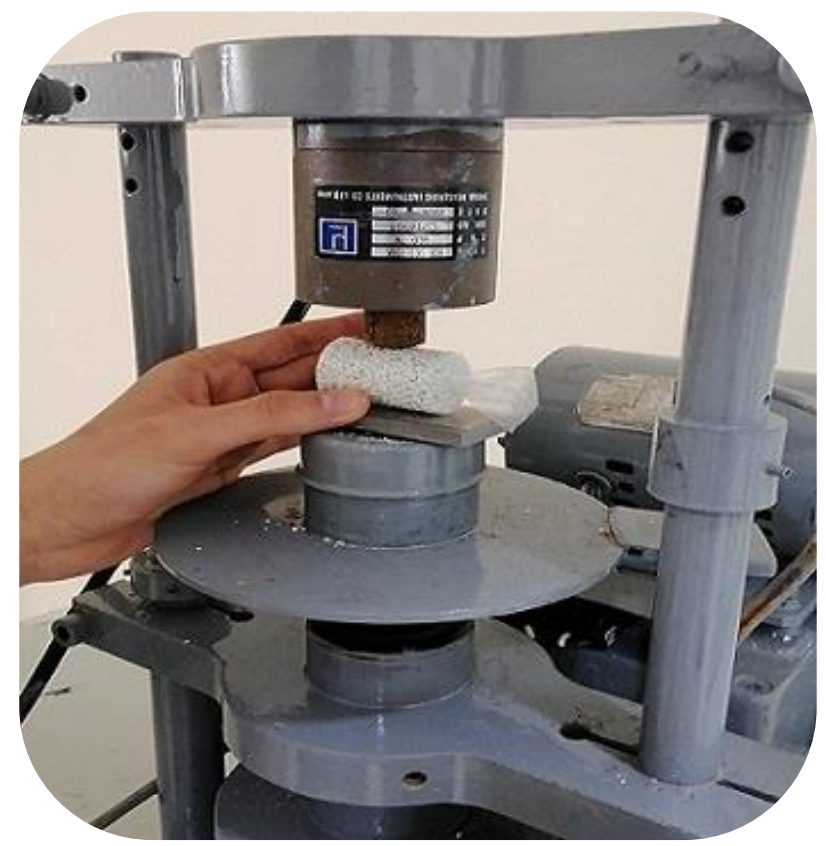

Figure 1. The cake strength evaluation using durometer machine

The principal objective of this part is to identify the crucial few anti-caking components that impact the cake strength of fertilizer. A Plackett-Burman screening design with 8 experiments for 7 components was recommended as the first step to identify the significant ones. The seven compounds chosen in the present investigation were tested between two levels: a high level (+1) and a low level
$(-1)$. The experimental design and the cake strength results are listed in Table 2. After data processing of screening design results, we got the estimated coefficients in the coded values using NemrodW software.

The following equation (Eq.2) represents the mathematical model gathered for the seven studied components:

$Y=30.4-1.9 X_{1}-0.9 X_{2}+0.8 X_{3}+1.2 X_{4}-1.7 X_{5}+1.9 X_{6}+1.7 X_{7}$ 
Table 2. Cake strength results of Plackett-Burman experimental design for seven parameters after the coating process.

\begin{tabular}{|c|c|c|c|c|c|c|c|c|}
\hline $\mathbf{R u n}$ & Glycerol & Molasses & $\mathrm{Na}_{2} \mathrm{SO}_{4}$ & $\mathrm{~K}_{2} \mathrm{SO}_{4}$ & $\mathrm{CaCO}_{3}$ & $\mathrm{Na}_{2} \mathrm{CO}_{3}$ & $\mathrm{SiO}_{2}$ & $\begin{array}{c}\text { Cake strength } \\
(\mathrm{kg})\end{array}$ \\
\hline $\mathbf{1}$ & 0.8 & 0.8 & 0.4 & 0.2 & 0.4 & 0.2 & 0.2 & 21.9 \\
\hline $\mathbf{2}$ & 0.4 & 0.8 & 0.4 & 0.4 & 0.2 & 0.4 & 0.2 & 35.3 \\
\hline $\mathbf{3}$ & 0.4 & 0.4 & 0.4 & 0.4 & 0.4 & 0.2 & 0.4 & 33.3 \\
\hline $\mathbf{4}$ & 0.8 & 0.4 & 0.2 & 0.4 & 0.4 & 0.4 & 0.2 & 28.3 \\
\hline $\mathbf{5}$ & 0.4 & 0.8 & 0.2 & 0.2 & 0.4 & 0.4 & 0.4 & 31.2 \\
\hline $\mathbf{6}$ & 0.8 & 0.4 & 0.4 & 0.2 & 0.2 & 0.4 & 0.4 & 34.3 \\
\hline $\mathbf{7}$ & 0.8 & 0.8 & 0.2 & 0.4 & 0.2 & 0.2 & 0.4 & 29.5 \\
\hline $\mathbf{8}$ & 0.4 & 0.4 & 0.2 & 0.2 & 0.2 & 0.2 & 0.2 & 29.1 \\
\hline
\end{tabular}

The above model presented the results on two graphs, as shown in Figure 2, 3. The Pareto chart (Figure 2) puts the variables to decrease the absolute magnitude of the effects, enabling the essential variables to be identified immediately. It can be represented graphically in the form of a normalized square of each effect $P_{i}$, where the function $P_{i}$ is defined as follows:

$P_{i}=\frac{b_{i}^{2}}{\sum_{i=1}^{7} b_{i}^{2}} \times 100$
On which $b_{i}$, are defined as the coefficients of the model (Eq.2).

Analysis of the Pareto chart (Figure 2) shows that the four first coefficients add up together to nearly $82 \%$ of the coefficients' total sums of squares. Therefore, sodium carbonate, glycerol, silicon dioxide, and calcium carbonate significantly affect the fertilizer's cake strength. Whereas potassium sulfate, sodium sulfate, and molasses don't seem to be active.

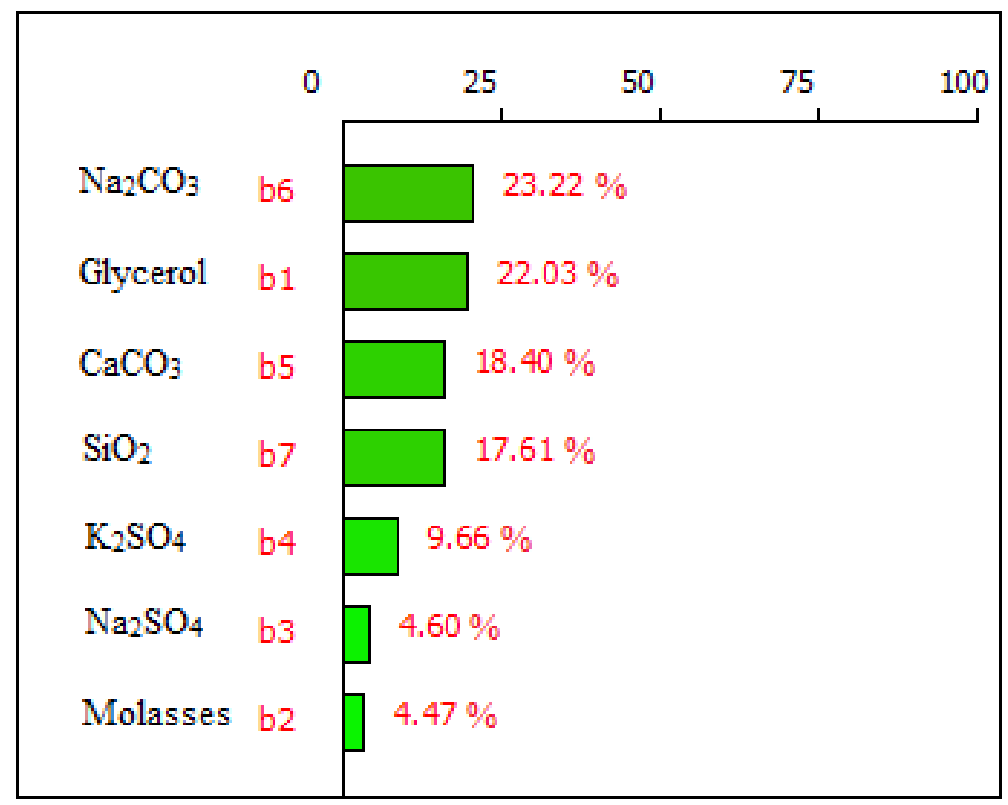

Figure 2. Pareto chart

The second graph is an effects plot (Figure 3), which shows the magnitude and sign of each effect in the variables' original order.

According to the effects graph (Figure 3), glycerol and calcium carbonate have a maximal negative effect on the cake strength. The more we add $\mathrm{CaCO}_{3}$ and glycerol, the more the cake strength decreases, and the coating is effective. However, sodium carbonate and silicon dioxide have a maximal positive effect. The more we add them, the more the cake strength increases, and the coating is not effective. 


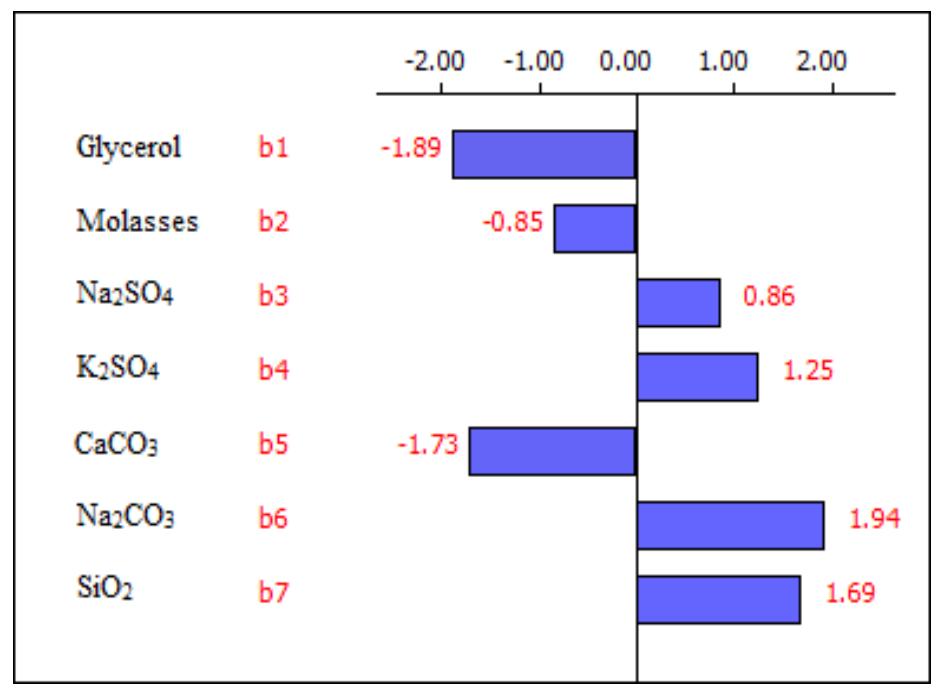

Figure 3. Effects graph

From these results, we selected only components that negatively affect glycerol and calcium carbonate as the main components to formulate a new anti-caking agent capable of reducing the cake strength of fertilizer in the presence of molasses as a technical choice. Molasses is a compound with a particular texture, sticky and very soluble in the soil. Moreover, it is a robust binder that allows fixing powders. Even its variation doesn't seem significant; we use it as one of the three factors used in the mixture design to improve the binding power of the mixture to adhere to the total weight of $\mathrm{CaCO}_{3}$ powder.

\subsection{Mixture experimental design}

Frequently, because of physical, theoretical, economic, and legislative reasons, some experimental optimization situations involving mixtures where the $X_{i}$ proportions are not allowed to vary from 0 to $100 \%$. Instead, some, or all, of the component proportions are restricted by either a lower bound and/or an upper bound.

In practice, we can introduce some lower limits (or bounds), some upper limits (or bounds), or some lower and upper limits on the proportions of the interest components. In the case of component $i$, these constraints may be written as:

$0<L_{i} \leq X_{i} \leq 1$

$$
\begin{aligned}
& 0 \leq X_{i} \leq U_{i}<1 \\
& 0<L_{i} \leq X_{i} \leq U_{i}<1
\end{aligned}
$$

Where $L_{i}$ is the lower bound and $U_{i}$ is the upper bound ${ }^{47}$.

When placing consistent lower or/and upper constraints on some or all component proportions, the experimental region resulting from the restrictions can still correspond to a regular simplex. Unfortunately, this is not always the case. The shape of the design region depends absolutely on the constraints chosen.

It must be noted that, even when the upper $\left(U_{i}\right)$ and/or the lower $\left(L_{i}\right)$ explicit bounds are considered in mixture experiments, indirectly, some implicit lower $\left(L_{i}^{*}\right)$ and/or upper $\left(U_{i} *\right)$ limits are also determined.

This study has chosen the three components gathered from the screening design to formulate anti-caking compositions to coat ternary granular fertilizer. This part aims to determine the composition of the mixture for which the cake strength is kept as low as possible.

The three components in the mixture are glycerol $\left(X_{I}\right)$, molasses $\left(X_{2}\right)$, and calcium carbonate $\left(X_{3}\right)$ with upper and lower constraints. Their variation ranges are mentioned in Table 3 below. Knowing that the sum of the three components $\left(X_{1}, X_{2}\right.$, and $\left.X_{3}\right)$ must be one, which is equivalent to $100 \%$.

Table 3. Variation ranges of different components in the mixture experimental design.

\begin{tabular}{|c|c|c|}
\hline Variables & Lower constraint & Upper constraint \\
\hline $\boldsymbol{X}_{\boldsymbol{I}}$ : Glycerol & 0.35 & 1.00 \\
\hline $\boldsymbol{X}_{\mathbf{2}}$ : Molasses & 0.00 & 0.65 \\
\hline $\boldsymbol{X}_{\mathbf{3}}$ : Calcium carbonate & 0.00 & 0.65 \\
\hline
\end{tabular}

The relationship between the cake strength and the mixture of the three components mentioned above can be defined using Scheffé's canonical special cubic model ${ }^{48}$ given by Eq.4:

$Y=\sum b_{\mathrm{i}} X_{\mathrm{i}}+\sum b_{i j} X_{i} X_{j}+\sum b_{i j k} X_{i} X_{j} X_{k}$ (Eq.4) 
As seen from Figure 4 below, the experimental domain that we got by imposing explicit upper and lower bound for glycerol gives us indirectly two implicit upper and lower bounds for molasses and $\mathrm{CaCO}_{3}$ to maintain the regular shape of an L-simplex.

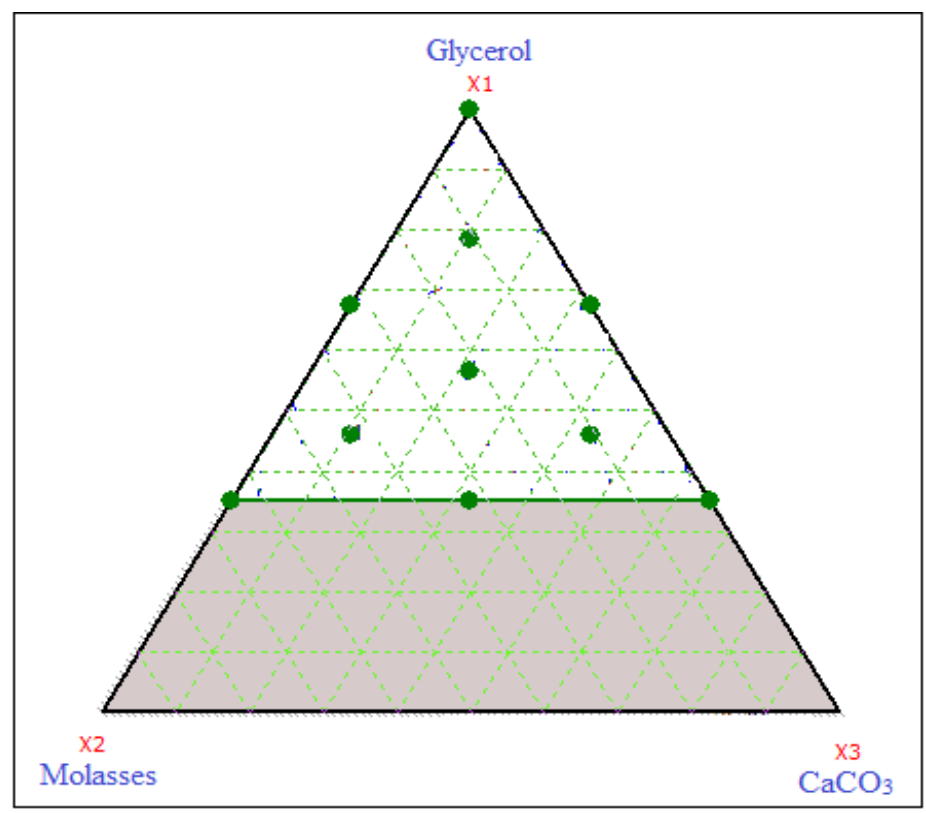

Figure 4. The experimental interest domain of the mixture design for glycerol, molasses, and $\mathrm{CaCO}_{3}$

The coating efficiency of the various formulations prepared will be performed on uncoated ternary granular fertilizer (NPK), a mean cake strength of $38.2 \mathrm{Kg}$. Evaluating the cake strength of all trials in the experiment design was done using a durometer machine.
Therefore, the experimental design selected is the augmented simplex-centroid design 49 with checkpoints, where each experiment is replicated at every design point of the simplex.

Table 4. Experimental conditions of the mixture design with the cake strength results.

\begin{tabular}{|c|c|c|c|c|}
\hline Run & Glycerol & Molasses & CaCO3 & Cake strength $($ Kg $)$ \\
\hline $\mathbf{1}$ & 1.000 & 0.000 & 0.000 & 16.8 \\
\hline $\mathbf{2}$ & 1.000 & 0.000 & 0.000 & 22.5 \\
\hline $\mathbf{4}$ & 0.350 & 0.650 & 0.000 & 23.7 \\
\hline $\mathbf{5}$ & 0.350 & 0.650 & 0.000 & 25.2 \\
\hline $\mathbf{6}$ & 0.350 & 0.000 & 0.650 & 25.6 \\
\hline $\mathbf{7}$ & 0.350 & 0.000 & 0.650 & 19.0 \\
\hline $\mathbf{8}$ & 0.675 & 0.325 & 0.000 & 20.7 \\
\hline $\mathbf{9}$ & 0.675 & 0.325 & 0.000 & 21.5 \\
\hline $\mathbf{1 0}$ & 0.675 & 0.000 & 0.325 & 20.0 \\
\hline $\mathbf{1 1}$ & 0.675 & 0.000 & 0.325 & 20.2 \\
\hline $\mathbf{1 3}$ & 0.350 & 0.325 & 0.325 & 22.0 \\
\hline $\mathbf{1 4}$ & 0.350 & 0.325 & 0.325 & 19.6 \\
\hline $\mathbf{1 5}$ & 0.566 & 0.217 & 0.217 & 19.3 \\
\hline
\end{tabular}


In Table 4, the design settings of the threecomponents used in the mixture design involve a total of 17 blends prepared to coat granules. Seven experiments that are replicated (runs n. 1-14) correspond to the vertices, the center of the sides, and the center of the domain. The remaining three experiments (runs n. 15-17) correspond to the checkpoints. The first 14 formulations have been used to build the predictive model, and the remaining 3 formulations (checkpoints) have been used to test the validity of the model in the explored domain. The experimental conditions of the mixture design and the cake strength results are listed in Table 4.

\subsubsection{Model validation}

Table 5. Validation of the model with the checkpoints method.

\begin{tabular}{|c|c|c|c|c|c|c|c|}
\hline Run & $\boldsymbol{Y}_{\text {exp }}$ & $\boldsymbol{Y}_{\text {cal }}$ & $\boldsymbol{Y}_{\boldsymbol{e x p}}-\boldsymbol{Y}_{\boldsymbol{c a l}}$ & $\boldsymbol{d}_{\boldsymbol{u}}$ & Standard deviation & $\boldsymbol{t}_{\text {exp }}$ & $\boldsymbol{P}$-value (significance) \\
\hline $\mathbf{1 5}$ & 19.300 & 19.307 & -0.007 & 0.224 & 0.953 & -0.008 & 0.994 \\
\hline $\mathbf{1 6}$ & 20.200 & 21.004 & -0.804 & 0.224 & 0.953 & -0.844 & 0.427 \\
\hline $\mathbf{1 7}$ & 20.300 & 22.305 & -2.005 & 0.224 & 0.953 & -2.103 & 0.074 \\
\hline
\end{tabular}

Table 5 clearly shows that the differences between measured and calculated responses are not statistically significant $(P$-value $\geq 0.05)$ when using the t-test at a $95 \%$ probability level. From these results, we can conclude that the model can be used to describe the cake strength of granules and predict it in the studied domain. The postulated model can be considered a good fit model due to its coefficient of determination $\mathrm{R}^{2}$ (0.91), which explains the studied phenomenon rightly; it's also the appropriate model for this study $\left(\mathrm{R}_{\text {adj }}^{2}=0.86\right)$.
The obtained fourteen cake strength results (runs n.1-14) given in Table 4 were used to estimate the model coefficients, and the results of the three checkpoints (runs n. 15-17) were deployed to test the validity of the model ${ }^{50-51}$. The checkpoints method consists of comparing the experimental responses of additional experiments located in the studied domain but not used to calculate the model coefficients, with the calculated responses given by the model ${ }^{51-52}$. The model can be considered as validated if, for each checkpoint, the difference between the predicted and the experimental response is not statistically significant ${ }^{47,53}$. The three checkpoints' cake strength results (runs n. 15-17) given in Table 5 were compared with the predicted responses to confirm that the difference is not statistically significant. Therefore, the model is validated.
$Y=16.7 X_{1}+23.1 X_{2}+25.2 X_{3}+0.01 X_{1} X_{2}+1.4 X_{1} X_{3}-17.1 X_{2} X_{3}+11.0 X_{1} X_{2} X_{3}$

\subsubsection{Graphical exploitation of the validated model}

In order to analyze the proportions variation effect of the three anti-caking components on the cake strength of fertilizer, the response trace technique was applied. The graph (Figure 5) represents the variation in the cake strength values by changing the percentage of a single component and keeping the relative percentages of the two other components fixed. The reference mixture is presented as point 0.00 on the horizontal axis. In Our case it corresponds to $57 \%$ glycerol $+21,5 \%$ molasses $+21,5 \% \mathrm{CaCO}_{3}$, because it is the center of the explored region (centroid).
Once the test is not statistically significant, the cake strength values obtained at the checkpoints can be incorporated to recalculate the model coefficients to make them more accurate. The information obtained from these points is also used ${ }^{47}$. The Scheffé's canonical special cubic model of the three studied components takes the following form (Eq. 5) based on the pseudo-components domain:
From the response trace plots (Figure 5), it can be noticed that the cake strength for the quantities of glycerol less than $57 \%$ takes constant and high values. However, from this point, it progressively decreases until it reaches its minimum. In other words, an increase in the concentration of glycerol leads to a remarkable reduction of the cake strength. A quiet augmentation of the cake strength can be observed with calcium carbonate, where an increase of the response can be explained by the low concentration of binder fixing the powder. Finally, the third and last component, as seen in the response trace plots, the cake strength is insensitive to the amount of molasses added. 


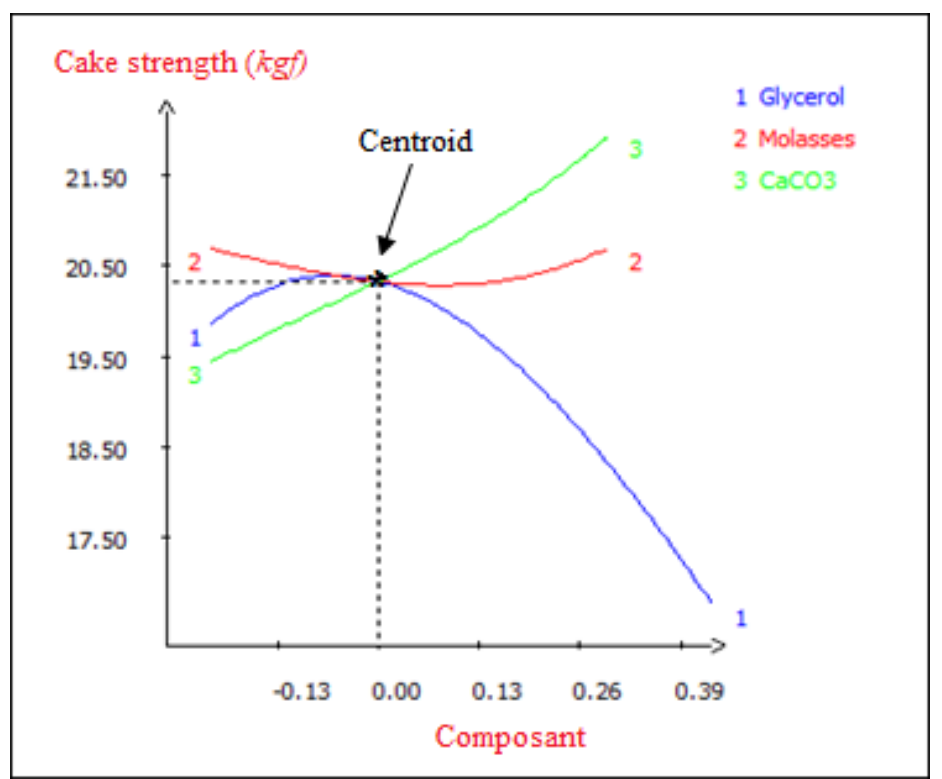

Figure 5. Response trace plot for the cake strength of ternary granular fertilizer

Looking at the estimated coefficients in the model (Eq.5), it doesn't seem easy to describe the cake strength of fertilizer, especially when the three components' proportions are varied simultaneously. For this reason, the graphical representation of the postulated model into a mixture contour plot makes the description feasible and more understandable ${ }^{54}$.
The contour plot below (Figure 6) presents an infinity of combinations between glycerol, molasses, and calcium carbonate. Different cake strength results are based on the interest response and the available raw materials.

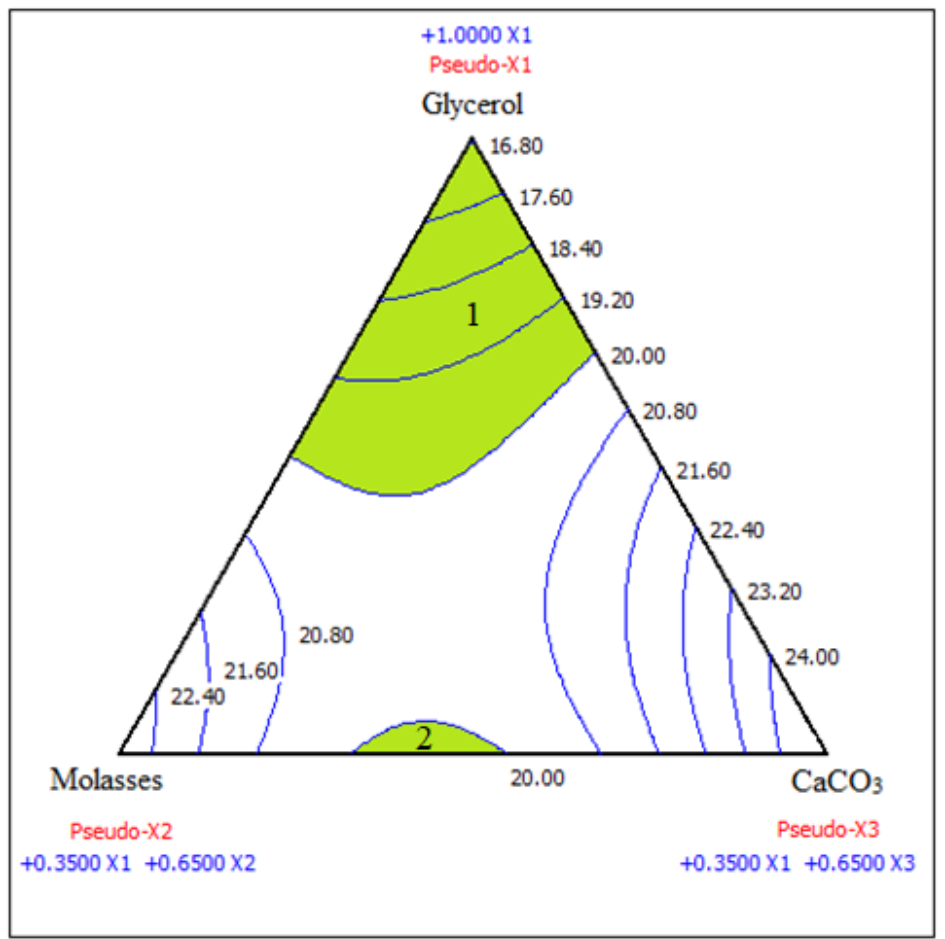

Figure 6. Contour plot for the cake strength of ternary granular fertilizer

The graph analysis clearly shows the effect of the proportions of three components on the studied response. In this study, we have to remember that we aim to formulate an anti-caking agent that can reduce the cake strength, ideally less than $20 \mathrm{kgf}$. The contour plot indicates that many blends will meet our requirement, especially those that are located at the contour green area (zone 1, 2).

These blends correspond to the ternary mixtures of glycerol, molasses, and $\mathrm{CaCO}_{3}$ in the zone rich in glycerol (zone 1). The lowest possible proportion of 
glycerol should be 0.8 related to the original space.

Glycerol is known to be hygroscopic, which gives it the ability to protect fertilizer granules from moisture. The center of the bottom side of the simplex (zone 2) corresponds to the optimum proportion of molasses to adhere to the total quantity of $\mathrm{CaCO}_{3}$ and provides a cake strength of fewer than $20 \mathrm{kgf}$. It must be underlined that this efficiency comes from the hygroscopic property of $\mathrm{CaCO}_{3}$, preventing the exchange of moisture between the granules and the external atmosphere. Therefore, the use of calcium carbonate in the presence of molasses (as a binder), even with less glycerol, reduces the cake strength of ternary granular fertilizer.

\section{Conclusion}

From the results obtained in this work, it can be concluded that the combination of two designs of the experiment: The Plackett-Burman design and mixture design, has been used effectively to select the significant components and to formulate anti-caking compositions capable of reducing the caking tendency of ternary granular fertilizer. The production of a predictive mathematical model based on glycerol performance, calcium carbonate, and molasses allowed us to understand how granules' cake strength is affected by the proportion's variation of different mixture component fractions to adjust compositions by the end-user expectations. These valuable results showed several formulations of the three-components that can reduce the cake strength of granules by up to $56 \%$ compared to uncoated fertilizer, which has significantly improved their flowability. Finally, we hope that the integration of chemometric tools like DoE helps fertilizer manufacturers further enhance their products' physical quality, where customer satisfaction is their ultimate goal.

\section{Acknowledgments}

This work was supported by the Research and Development initiative of OCP GROUP. Call for projects about phosphate (APHOS)-sponsored by OCP (OCP Foundation, R\&D OCP, Mohammed VI Polytechnic University, National Center of Scientific and Technical Research CNRST, Ministry of Higher Education, Scientific Research and Professional Training of Morocco (MESRSFC). The project entitled: Development of OCP additives for improving the physical quality of fertilizers, project ID *VAL-ELS-01/2017*.

We would like to thank all the staff members of the fertilizer unit of the Research and Development department at Jorf lasfar (Industrial Site of OCP GROUP).

\section{References}

1- D. Turner, C. Batello, H. Dreyer, T. Calles, World fertilizer trends and outlook to 2022, FAO, Rome, 2019.

2- M. Prud'homme, J. de Sousa, V. Couturier, O. Rousseau, S. Marcel-Monnier, Fertilizer outlook 2018-2022, IFA, Berlin, 2018.

3- G. S. Hunter, Dry fertilizer compositions and method for making the same, U.S. Pat. Appl. 6558445 B2, 2003.

4- T. M. Lowry, F. C. Hemmings, The properties of powders. Part I. The caking of salts, J. Soc. Chem. Ind., 1920, 39, 101-110.

5- J. R. Adams, A. R. Merz, Hygroscopicity of fertilizer materials and mixtures, Ind. Eng. Chem., 1929, 21, 305-307.

6- A. Tyc, D. Niewes, S. Penkala, R. Grzesik, K. Hoffmann, J. Hoffmann, Influence of anticaking agents on the highly effective organic coatings for preventing the caking of ammonium nitrate fertilizers, coating, 2020, 10, 1093.

7- M. Chen, S. Wu, S. Xu, B. Yu, M. Shilbayeh, Y. Liu, X. Zhu, J. Wang, J. Gong, Caking of crystals: Characterization, mechanisms and prevention, Powder Technol., 2018, 337, 51-67.

8- U. Zafar, V. Vivacqua, G. Calvert, M. Ghadiri, J. A. S. Cleaver, A review of bulk powder caking, Powder Technol., 2017, 313, 389-401.

9- T. Ahlnäs, T. Löfgren, T. Kankaanpää, An anticaking agent, Eur. Pat. Appl. 0858439 B1, 2002.

10-A. A. A. Bod, M. Verschuren, M. Jansen, S. Jiang, J. A. M. Meijer, W. J. P. van Enckevort, E. Vlieg, Influence of anti-caking agents on the caking of sodium chloride at the powder and twocrystal scale, Powder Technol., 2015, 277, 262-267.

11-M. Schultz, C. Laschilier, R. Adams, J. Hancock, M. Johnson, K. L. Jacobson, D. Fuchs, K. Rinas, Coatings and additives containing fatty acids, salts of fatty acids, or similar compounds, for use as de-dust and/or anti-caking agents for granular products, U.S. Pat. Appl. 20160304410 A1, 2016.

12-D. T. Parker, M. Ogzewalla, Z. T. Burrell, Fertilizer coating for dust control and / or anticaking, U.S. Pat. Appl. 20190077723 A1, 2019.

13-S. Gadman, J. Rodsvic, Method for coating particulated fertilizers, W.O. Pat. Appl. 9915480 A1, 1999.

14-T. Löfgren, O. Grönfors, T. Ahlnäs, Method for the coating of particles, the use of a coating suspension and a coated product, Eur. Pat. Appl. 1194220 B1, 2004.

15-T. Wasvik, E. F. Hansen, S. G. Snartland, Agricultural composition and conditioning agent for reducing hygroscopicity, dust formation and caking of fertilizers, W.O. Pat. Appl. 0138263 A1, 2001. 
16-E. F. Hansen, T. Wasvik, S. G. Snartland, Agricultural composition and conditioning agent, Eur. Pat. Appl. 1390322 B1, 2006.

17-H. Hero, M. Urhonen, J. Niskala, Particulate fertilizer product, method for its preparation and use, Eur. Pat. Appl. 1861342 B1, 2014.

18 -S. Spence, Anti-caking and dust control coating compositions containing liquid-dispersed metallic salts of fatty acids and methods of using same, Eur. Pat. Appl. 1627865 A1, 2006.

19-K. J. Wheeler, R. M. Young, G. M. Harman, M. W. Hill, Coating for improved granular fertilizer efficiency, U.S. Pat. Appl. 9938202 B2, 2018.

20-X. He, J. Li, M. Chen, Y. Jin, Y. Wang, J. Li, Resistance of deliquescence and caking to enhance the effective utilization of potassium nitrate: A novel surface modification method by SDS, Powder Technol., 2019, 356, 500-507.

21-A. M. M. de Laat, B. M. de Roode, T. W. L. Brooijmans, Formulation aid, U.S. Pat. Appl. 20190161814 A1, 2019.

22-J. F. Ang, J. F. Pond, M. Wang, L. A. McKee, Carbonate-based anti-caking agent with reduced gas release properties, U.S. Pat. Appl. 20040109927 A1, 2004.

23-R. A. Lipasek, L. S. Taylor, L. J. Mauer, Effects of anti-caking agents and relative humidity on the physical and chemical stability of powdered vitamin C, J. Food Sci., 2011, 76, 1062-1074.

24-R. A. Lipasek, J. C. Ortiz, L. S. Taylor, L. J. Mauer, Effects of anti-caking agents and storage conditions on the moisture sorption, caking, and flowability of deliquescent ingredients, Food Res. Int., 2012, 45, 369-380.

25-R. G. Brereton, Chemometrics: Data Analysis for the Laboratory and Chemical Plant, Wiley, Chichester, 2003, 15-19.

26-R. D. Snee, Experiements with mixtures, Chemtech, Nov., 1979, 702-710.

27-D. Mathieu, J. Nony, R. Phan-Tan-Luu, Nemrodw (V-2007), L.P.R.A.I, Marseille, France, 2007. (www.nemrodw.com).

28-A. El Agri, A. Hafnaoui, M. Khouloud, M. Lakehal Senhaji, M. EL Asri, A. Meliani, The use of experimental design methodology in the formulation of a new additive for the dispersion of elemental sulfur in triple superphosphate fertilizer, Mediterr. J. Chem., 2020, 10, 595-607.

29-C. P. Santos, T. J. Rato, M. S. Reis, Design of Experiments: A comparison study from the nonexpert user's perspective, J. Chemom., 2019, 33, e3087.

30-L. Makouki, K. Tarbaoui, S. Glissi, S. Mansouri, H. Hannache, M. Oumam, Applying response surface methodology to optimize the decarbonization process of Timahdit oil shale, Mediterr. J. Chem., 2019, 8, 372-379.

31-J. Goupy, L. Creighton, Introduction aux plans d'expériences (3e édition), Dunod, Paris, 2006, 31-202.
32-T. Lundstedt, E. Seifert, L. Abramo, B. Thelin, A. Nystrom, J. Pettersen, R. Bergman, Experimental design and optimization, Chemom. Intell. Lab. Syst., 1998, 42, 3-40.

33-R. L. Plackett, J. P. Burman, The design of optimum multifactorial experiments, Biometrika, 1946, 33, 305-325.

34-Analytical Methods Committee, AMCTB No 55, Experimental design and optimisation (4): Plackett-Burman designs, Anal. Methods., 2013, 5, 1901-1903.

35-T. D. Pham, N. K. Nguyen, C. M. Tran, M. P. Vuong, Constructing response surface designs with orthogonal quadratic effects using cyclic generators, Chemom. Intell. Lab. Syst., 2020, 198, 103918.

36-F. Rossi, V. Mirtchev, Mixture experimental design, statistics for food scientists, Elsevier Inc., San Diego, 2016, 149-167.

37-M. A. Bezerra, V. A. Lemos, C. G. Novaes, R.M. de Jesus, H. R. S. Filho, S. A. Araújo, J. P. S. Alves, Application of mixture design in analytical chemistry, Microchem. J., 2020, 152, 104336.

38-Z. Jeirani, B. Mohamed Jan, B. Si Ali, I. Mohd. Noor, S. Chun Hwa, W. Saphanuchart, The optimal mixture design of experiments: Alternative method in optimizing the aqueous phase composition of a microemulsion, Chemom. Intell. Lab. Syst., 2012, 112, 1-7.

39-M. L. Ntsoane, D. Sivakumar, P. V. Mahajan, Optimisation of $\mathrm{O}_{2}$ and $\mathrm{CO}_{2}$ concentrations to retain quality and prolong shelf life of "shelly" mango fruit using a simplex lattice mixture design, Biosyst. Eng. 2020, 192, 14-23.

40-G. G. Marcheafavea, C. D. Tormenaa, E. Daiane Pauli, M. Rakocevic, R. E. Bruns, I. S. Scarminio, Experimental mixture design solvent effects on pigment extraction and antioxidant activity from Coffea arabica L. leaves, Microchem. J., 2019, 146, 713-721.

41-Y. Liu, M. Q. Liu, Construction of uniform designs for mixture experiments with complex constraints, Commun. Stat. Theory Methods, 2016, 45, 2172-2180.

42-S. Cafaggi, R. Leardi, B. Parodi, G. Caviglioli, G. Bignardi, An example of application of a mixture design with constraints to a pharmaceutical formulation, Chemom. Intell. Lab. Syst., 2003, 65, 139-147.

43-D. N. T. Hay, Composition for dust control and improved material handling, U.S. Pat. Appl. 9505965 B2, 2016.

44-B. L. Tran, S. Bhattacharja, S. J. Blubaugh, Methods and compositions for dust control and freeze control, U.S. Pat. Appl. 7398935 B2, 2008.

45-R. F. Buchholz, D. W. Quinn, Particulate fertilizer dust control, U.S. Pat. Appl. 5360465 A1, 1994.

46-F. E. Sexton, J. A. Gratz, K. E. Cox, Combination ammonium sulfate/drift reducing 
adjuvant and wet bond process for making the same, U.S. Pat. Appl. 6878180 B2, 2005.

47-S. D. Brown, R. Tauler, B. Walczak, Experimental Design for Mixture Studies, Comprehensive Chemometrics: Chemical and Biochemical Data Analysis, Elsevier, 2009, 391-449.

48-H. Scheffé, Experiments with Mixtures, J. R. Stat. Soc., 1958, 20, 344-360.

49-H. Scheffé, The Simplex-Centroid Design for Experiments with Mixtures, J. R. Stat. Soc., 1963, 25, 235-251.

50-R. D. Snee, Developing Blending Models for Gasoline and Other Mixtures, Technometrics, 1981, 23, 119-130.

51-J. T. Shelton, A. I. Khuri, J. A. Cornell, Selecting Check Points for Testing Lack of Fit in Response
Surface Models, Technometrics, 1983, 25, 357-365.

52-R. Huisman, H. V. Van Kamp, J. W. Weyland, D. A. Doornbos, G. K. Bolhuis, C. F. Lerk, Development and optimization of pharmaceutical formulations using a simplex lattice design, Pharm. Weekblad Sci., 1984, 6, 185-194.

53-A. Khaskhoussi, L. Calabrese, H. Bouhamed, A. Kamoun, E. Proverbio, J. Bouaziz, Mixture design approach to optimize the performance of $\mathrm{TiO}_{2}$ modified zirconia/alumina sintered ceramics, Mater. Des., 2018, 137, 1-8.

54-G. Geoffrey vining, J. A. cornell, R. H. Myers, A Graphical Approach for Evaluating Mixture Designs, J. R. Stat. Soc. Ser. C (Appl. Stat.) 1993, 42, 127-138. 